\title{
Síndrome de Boerhaave: reporte de un caso y revisión de la literatura
}

\author{
Boerhaave syndrome. Case report and literature review \\ Ibzan J. Salvador-Ibarra* y Alejandro Pizaña-Davila \\ Departamento de Terapia Intensiva, Hospital Ángeles Mocel, Ciudad de México, México
}

\begin{abstract}
Resumen
Las lesiones espontáneas pueden afectar solo una parte de la pared esofágica (síndrome de Mallory-Weiss) o constituir una rotura de espesor total del órgano, dando lugar al síndrome de Boerhaave. Afecta con mayor frecuencia a los varones entre 50 y 70 años de edad. Clínicamente conforma la tríada de Mackler: vómito, dolor torácico intenso y enfisema subcutáneo cervical. El retraso en el diagnóstico explica su alta tasa de mortalidad, de hasta el 40-60\% en los pacientes tratados a las 48 horas. El pronóstico mejora si se logra instaurar el tratamiento dentro de las primeras 24 horas.
\end{abstract}

Palabras clave: Pared esofágica. Síndrome de Boerhaave. Tríada de Mackler. Vómito. Esofagografía.

\begin{abstract}
Spontaneous lesions can affect only a part of the esophageal wall (Mallory-Weiss syndrome) or constitute a full-thickness rupture of the organ, leading to Boerhaave syndrome. Most commonly affecting males between 50 and 70 years of age, Clinically, the Mackler triad is vomiting, severe chest pain, and subcutaneous cervical emphysema. The delay in diagnosis explains the high mortality rate of this pathology up to $40-60 \%$ in those treated at 48 hours. The prognosis improves if treatment is established within the first 24 hours.
\end{abstract}

Key words: Esophageal wall. Boerhaave syndrome. Mackler's triad. Vomiting. Esophagography.

\section{Introducción}

Las perforaciones esofágicas pueden ser iatrogénicas, traumáticas, por cuerpos extraños, relacionadas con otras patologías o espontáneas. Las lesiones espontáneas pueden afectar solo una parte de la pared esofágica (síndrome de Mallory-Weiss) o constituir una rotura de espesor total del órgano, dando lugar al síndrome de Boerhaave, que representa el $8-56 \%$ de todas las perforaciones esofágicas ${ }^{1}$. La primera descripción de una lesión emetógena del esófago se atribuye al cirujano holandés Hermann Boerhaave. Su informe del paciente y la lesión estaba contenido en una monografía de 70 páginas publicada en 1724. Su famoso paciente fue el Gran Almirante de los Países Bajos, el barón Von Wassenauer, quien presentó un dolor de pecho insoportable repentino

\section{Correspondencia:}

*Ibzan Salvador Ibarra

Gregorio V. Gelati, 29

Col. San Miguel Chapultepec I Secc, Miguel Hidalgo

C.P. 11850, Ciudad de México, México

Fecha de recepción: 06-01-2021

Fecha de aceptación: 11-03-2021

E-mail: ibzjah@gmail.com
Cir Cir. 2021;89(S2):26-30

Contents available at PubMed www.cirugiaycirujanos.com
la licencia CC BY-NC-ND 0009-7411/@ 2021 Academia Mexicana de Cirug
(http://creativecommons.org/licenses/by-nc-nd/4.0/). 
posterior a una emesis autoinducida. Cuando poco tiempo después murió, el Dr. Boerhaave realizó una autopsia y encontró la perforación esofágica que ahora lleva su nombre?

La rotura esofágica espontánea, conocida como síndrome de Boerhaave, es poco frecuente, pero tiene una gran mortalidad. Muchas veces se manifiesta solo como un dolor abdominal ${ }^{3}$. Independientemente del mecanismo de acción, la rotura esofágica se considera la más grave del tracto digestivo $0^{4,5}$, con una mortalidad superior al $30 \%{ }^{6}$. El retraso en el diagnóstico ocurre hasta en un $50 \%$ de los pacientes ${ }^{7}$. El diagnóstico temprano suele estar entorpecido por la amplitud y la mayor prevalencia de los diagnósticos diferenciales, entre los que se encuentran la úlcera gástrica o duodenal perforada, el infarto agudo de miocardio, la pericarditis, el neumotórax, la tromboembolia pulmonar, la hernia diafragmática, el aneurisma disecante de la aorta y la pancreatitis aguda ${ }^{8}$.

\section{Caso clínico}

Varón de 73 años sin antecedentes de importancia que acude al servicio de urgencias, por su propio pie, por un cuadro de 5 horas de evolución que inició tras la ingesta de una comida copiosa. Presenta sensación de plenitud posprandial y posteriormente vómito gastroalimentario en cinco ocasiones, con posterior dolor en la región retroesternal, intenso (8/10 en la escala analógica visual), opresivo, con irradiación hacia la espalda, y disnea progresiva.

En la exploración física el paciente se encuentra despierto, escala de Glasgow 15 puntos, facies álgica, polipneico, con enfisema subcutáneo en la región supraclavicular y el tórax superior bilateral con crepitación, sin equimosis, con requerimiento de oxígeno suplementario por puntas nasales a $5 \mathrm{l} / \mathrm{min}$, con disminución del murmullo vesicular en la región basal del hemitórax derecho y dolor abdominal a la palpación superficial y profunda en la región epigástrica, así como intolerancia al decúbito supino. El resto de la exploración no mostraba alteraciones, con los siguientes signos vitales: presión arterial 130/80 mmHg, frecuencia cardiaca 100 latidos por minuto, frecuencia respiratoria 20 respiraciones por minuto, temperatura $36.6^{\circ} \mathrm{C}$, saturación de oxígeno $96 \%$ y fracción inspirada de oxígeno $30 \%$. Los parámetros de laboratorio fueron: hemoglobina $15.7 \mathrm{~g} / \mathrm{dl}$, hematocrito $45.9 \%$, leucocitos $12.3 \times 10^{3} / \mu \mathrm{l}$, glucosa $128 \mathrm{mg} / \mathrm{dl}$, procalcitonina $34 \mathrm{ng} / \mathrm{ml}$ y troponina $\mathrm{I}<0,04 \mathrm{ng} / \mathrm{ml}$. El electrocardiograma mostró ritmo sinusal, frecuencia cardiaca

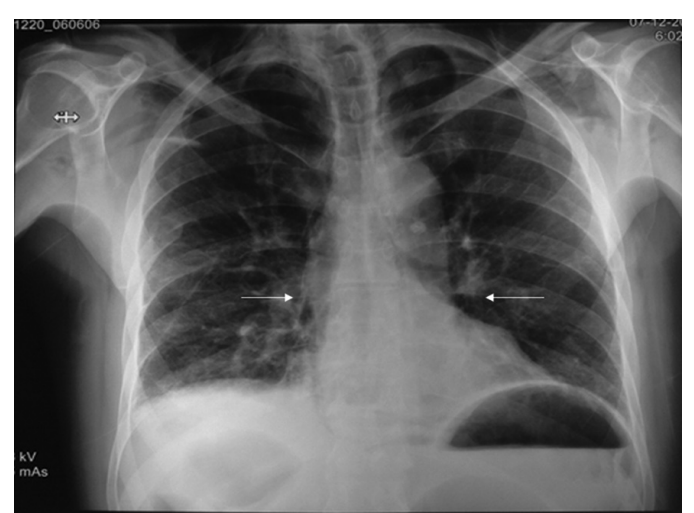

Figura 1. Radiografía de tórax posteroanterior en la que se observa un enfisema subcutáneo en el cuello y la región supraclavicular bilateral, así como ensanchamiento del mediastino y dilatación ventricular (flechas).

97 latidos por minuto, AQRS $0^{\circ}$, QRS $0.12 \mathrm{~s}$ y $\sin$ alteraciones del segmento ST. Se decidió realizar una radiografía de tórax, en la que se observó un enfisema subcutáneo en la región supraclavicular y ensanchamiento del mediastino (Fig. 1), y una tomografía computarizada de tórax con medio de contraste oral hidrosoluble, que confirmó el enfisema subcutáneo con extensión hasta la región escapular bilateral. También se observó neumomediastino bilateral, neumopericardio y fuga del medio de contraste hidrosoluble a nivel del tercio inferior del esófago hacia el mediastino medio, la cavidad pleural y el pericardio (Figs. 2 a 4). Se inició antibioticoterapia intravenosa (betalactámico y clindamicina).

Por la localización de la perforación en el tercio inferior del esófago y la fuga del medio de contraste hacia el retroperitoneo se decidió someter al paciente, a las 4 horas de ingreso, a una laparoscopia para drenaje del líquido pleural, mediastinal y retroperitoneal observado en los estudios de imagen, así como a cierre primario con funduplicatura. El paciente ingresó a quirófano para laparoscopía, en la cual se localizó el hiato esofágico, donde se observó en su cara anterior una zona de mucosa irritada y se realizó disección del ligamento esofagofrénico, identificando los pilares y liberando los vasos cortos. Posteriormente se realizaron ventana retroesofágica y disección de hiato esofágico, donde se observó una perforación de la pleura derecha con presencia de abundante contenido gástrico en la pleura y el mediastino, y se identificó la zona de perforación en el tercio inferior de la cara lateral derecha del esófago, de $1.5 \mathrm{~cm}$ de longitud, que se cerró con sutura y pegamento. 


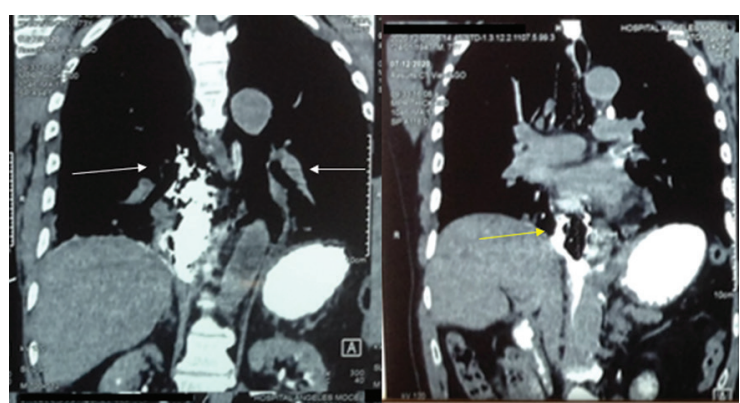

Figura 2. Tomografía computarizada de tórax, ventana para mediastino (corte coronal), con medio de contraste hidrosoluble, en la que se observa neumomediastino bilateral de predominio a nivel medio y posterior (flechas blancas), el cual no desplaza ni comprime las estructuras adyacentes. Se observa neumopericardio (flecha amarilla).
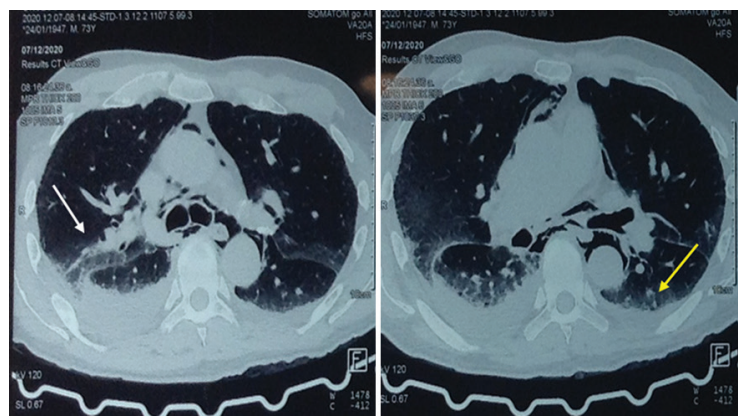

Figura 3. Tomografía computarizada de tórax, ventana pulmonar (corte transversal), en la que se observan una zona de mayor densidad en el segmento posterior del lóbulo inferior derecho(flecha blanca) y derrame pleural bilateral de predominio derecho con ocupación aproximada del 20\% (flecha amarilla).

A continuación se realizó funduplicatura tipo Dor y se colocaron drenajes en hiato esofágico y subdiafragmático izquierdo, y sonda endopleural en el espacio pleural derecho. Al egreso del quirófano, el paciente ingresó a la unidad de terapia intensiva por choque séptico, con presión arterial de $80 / 50 \mathrm{mmHg}$, frecuencia cardiaca de 110 latidos por minuto, frecuencia respiratoria de 18 respiraciones por minuto, temperatura de $37.0^{\circ} \mathrm{C}$, saturación de oxígeno del $94 \%$, fracción inspirada de oxígeno del $80 \%$ y llenado capilar de $5 \mathrm{~s}$, requiriendo manejo vasopresor con norepinefrina a dosis de $0.19 \mu \mathrm{g} / \mathrm{kg} / \mathrm{min}$ y vasopresina a dosis de $0.03 \mathrm{U} / \mathrm{min}$, así como ventilación mecánica invasiva y nutrición parenteral (Fig. 5). Los resultados de los cultivos reportaron crecimiento de Candida glabrata sensible al voriconazol $(\leq 0.12 \mu \mathrm{g} / \mathrm{ml})$, por lo que se realizó escalamiento terapéutico y se logró la extubación a las 72 horas del ingreso a la unidad de terapia intensiva. Se realizó esofagograma de control

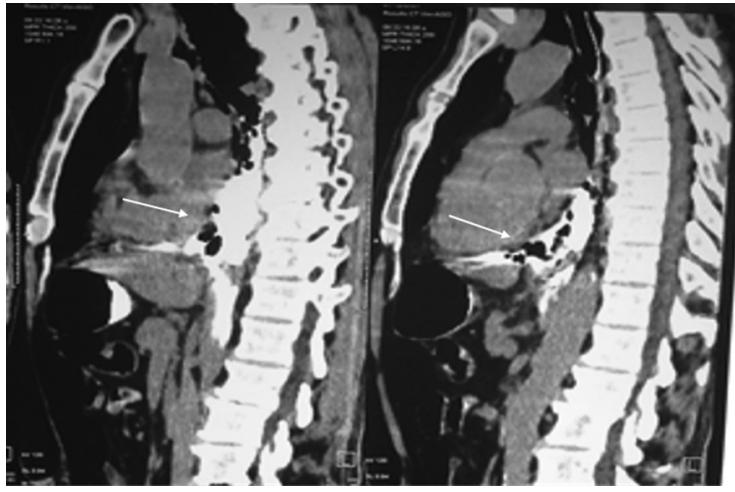

Figura 4. Tomografía computarizada de tórax (corte sagital) con medio de contraste hidrosoluble que muestra neumomediastino medio y posterior, y fuga del medio de contraste por el hiato aórtico hacia el retroperitoneo (flecha blanca).

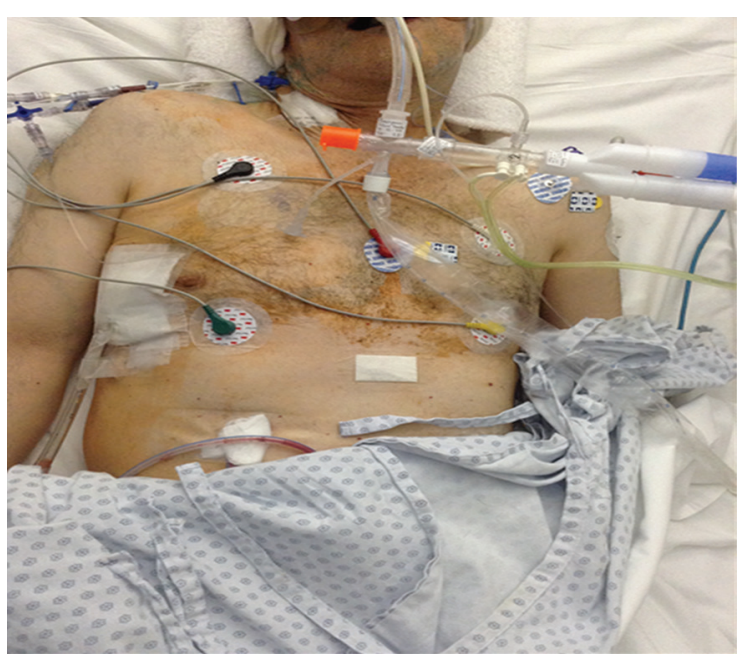

Figura 5. Paciente en la unidad de terapia intensiva por choque séptico. Se observa el enfisema subcutáneo en la región superior del tórax, bilateral.

a los 5 días del procedimiento quirúrgico y no se observó evidencia de fuga esofágica (Fig. 6). El paciente tuvo una evolución favorable, con resolución del cuadro de sepsis, reinicio y tolerancia de la vía oral, y egreso a los 10 días de ingreso hospitalario, con seguimiento posterior con estudio de imagen de control por consulta externa.

\section{Discusión}

La rotura espontánea del esófago se define como una dehiscencia de la pared esofágica en una zona macroscópicamente sana, en general como consecuencia de un aumento brusco de la presión 


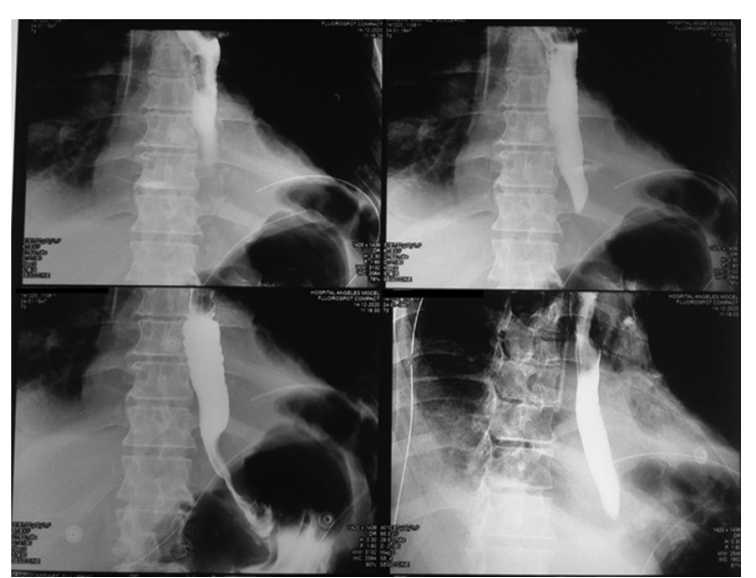

Figura 6. Esofagograma de control a los 5 días, en el que se observa la zona de pleurostomía en el hemitórax derecho, sin evidencia de fuga esofágica y con tránsitos bucofaríngeo y faringoesofágica normales.

esofágica combinado con una presión intratorácica negativa causada por el vómito 0 un esfuerzo $0^{\circ}$. Los datos obtenidos de cadáveres frescos con la porción medial del esófago pinzada han revelado que una presión de $150 \mathrm{mmHg}$ puede producir un desgarro de la unión gastroesofágica ${ }^{10}$. La porción intratorácica inferior del esófago es la parte más frágil del órgano, ya que es relativamente pobre en fibras musculares longitudinales y no está protegida por estructuras anatómicas; además, la presencia de un gran número de estructuras vasculares y nerviosas debilita la pared ${ }^{11}$. La perforación esofágica completa se produce en la pared lateral izquierda en el tercio inferior, 3-5 cm por arriba de la unión gastroesofágica del esófago distal, en el $90 \%$ de los casos $^{12-14}$. En ello influyen varios factores anatómicos, como el estrechamiento del músculo a nivel del esófago distal, el debilitamiento de esta pared por la entrada de vasos sanguíneos y nervios, la falta de apoyo de las estructuras circundantes y el hecho de que el esófago está inclinado hacia adelante a nivel del pilar diafragmático izquierdo ${ }^{15}$. La longitud de la perforación varía de 0.75 a $7.5 \mathrm{~cm}^{16,17}$. En el $80 \%$ de los casos hay comunicación con el espacio pleural, por lo que los contenidos del estómago y del esófago se desplazan hacia el tórax por la presión negativa ${ }^{18}$.

Afecta con mayor frecuencia a los varones entre 50 y 70 años de edad ${ }^{5}$, y presenta una gran asociación con el consumo de alcohol y la enfermedad por reflujo gastroesofágico ${ }^{19}$. Otros factores precipitantes son tos intensa, asma aguda, epilepsia, estreñimiento, parto, levantamiento de pesas, traumatismos toracoabdominales y maniobras de endoscopia ${ }^{19,20}$.
Clínicamente conforma la tríada de Mackler (vómito, dolor torácico intenso y enfisema subcutáneo cervical), pero rara vez esta se presenta de forma completa, siendo los síntomas principales vómito, dolor torácico (el más frecuente, presente en el $70 \%$ de los pacientes), disnea, disfagia, enfisema subcutáneo, taquicardia, fiebre, taquipnea y dolor epigástrico. En casos raros la perforación esofágica puede manifestarse por hematemesis u otros signos de hemorragia gastrointestinal, incluyendo la melena ${ }^{21,22}$.

El retraso en el diagnóstico explica la alta tasa de mortalidad de esta patología: del $10-25 \%$ en los casos tratados dentro de las primeras 24 horas hasta del 40-60\% en los tratados a las 48 horas $^{21}$. El pronóstico mejora si se logra instaurar el tratamiento dentro de las primeras 24 horas $^{18}$.

La radiografía de tórax muestra alteraciones en el $80-90 \%$ de los casos, con derrame pleural de predominio izquierdo, neumotórax, enfisema subcutáneo y neumomediastino. El estudio tomográfico permite un análisis más detallado del mediastino, evidenciar los abscesos adyacentes a la pleura, el esófago o el mediastino, y detectar la comunicación o la presencia de aire entre el esófago y el mediastino. La prueba diagnóstica de referencia ante la sospecha de una rotura esofágica es la esofagografía con contraste hidrosoluble, que aporta información sobre el tamaño y la localización de la perforación; además, es de gran utilidad en la planificación del abordaje quirúrgico ${ }^{23-25}$.

El tratamiento conservador está indicado en los pacientes hemodinámicamente estables con perforaciones contenidas y sin signos de sepsis. Incluye la colocación de un stent tubotorácico, gastrostomía o yeyunostomía de alimentación. Las opciones quirúrgicas son cierre primario, cierre primario reforzado (músculo intercostal, pleura, diafragma, estómago, epiplón y pulmón) y exclusión o resección esofágica. El cierre primario con refuerzo es el tratamiento de elección cuando el tiempo de evolución es menor de 24 horas. En casos de perforaciones largas, contaminaciones extensas, enfermedad esofágica asociada, fallo del cierre primario o sepsis persistente, están indicados procedimientos más agresivos, como exclusión y resección esofágica ${ }^{26}$.

\section{Conclusiones}

La perforación o rotura esofágica representa un desafío diagnóstico y terapéutico. El reconocimiento oportuno respaldado por imágenes radiológicas y el tratamiento con antibioticoterapia de amplio espectro, 
la realización de cultivos y, principalmente, el abordaje quirúrgico temprano, son determinantes pronósticos importantes. La elección del tratamiento depende del estado, el sitio, la extensión y la duración de la fuga, así como de la presencia de sepsis. Es importante considerar su diagnóstico ante cualquier presentación de dolor precordial y evento previo de vómito, el cual puede ser el factor desencadenante, como en el caso presentado.

\section{Agradecimientos}

A todo el personal que labora en la terapia intensiva del Hospital Ángeles Mocel.

\section{Responsabilidades éticas}

Protección de personas y animales. Los autores declaran que para esta investigación no se han realizado experimentos en seres humanos ni en animales.

Confidencialidad de los datos. Los autores declaran que en este artículo no aparecen datos de pacientes.

Derecho a la privacidad y consentimiento informado. Los autores han obtenido el consentimiento informado de los pacientes y/o sujetos referidos en el artículo. Este documento obra en poder del autor de correspondencia.

\section{Conflicto de intereses}

Los autores declaran no tener ningún conflicto de intereses.

\section{Financiamiento}

Los autores declaran no haber recibido ningún tipo de financiamiento para la realización de este artículo.

\section{Bibliografía}

1. Chirica M, Champault A, Dray X, Sulpice L, Munoz-Bongrnad N, Sarfati E, et al. Esophageal perforations. J Visc Surg. 2010;147:117-28.
2. Derbes VJ, Mitchell Jr RE. Hermann Boerhaave's Atrocis, nec descripti prius, morbi historia, the first translation of the classic case report of rupture of the esophagus, with annotations. Bull Med Libr Assoc. 1955;43:217-40.

3. De Schipper JP, Pull ter Gunne AF, Oostvogel HJM, Van Laarhoven CJHM. Spontaneous rupture of the oesophagus: Boerhaave's syndrome in 2008. Literature review and treatment algorithm. Dig Surg. 2009;26:1-6.

4. Ando H, Shitara Y, Hara K, Mogami Y, Kobayashi T, Yajima T, et al. Successful surgical treatment of a spontaneous rupture of the esophagus diagnosed two days after onset. Case Rep Gastroenterol. 2012;6:260-5.

5. Tonolini M, Bianco R. Spontanuous esophageal perforation (Boerhaave syndrome): diagnosis with CT-esophagography. J Emerg Trauma Shock. 2013;6:58-60.

6. Ríos Zambulido A, Martínez de Haro LF, Ortiz Escandell MA, Durán H, Munitiz Ruiz V, Parrilla Paricio P. Perforaciones esofágicas. Presentación de 23 casos. Gastroenterol Hepatol. 2000;23:379-83.

7. Tamatey MN, Sereboe LA, Tettey MM, Entusa-Mensah K, Gyan B. Boerhaave's syndrome: diagnosis and successful primary repair one month after the oesophageal perforation. Ghana Med J. 2013;47:53-5.

8. Teh E, Edwards J, Duffy J, Beggs D. Boerhaaver's syndrome: a review of management and outcome. Interact Cardiovasc Thorac Surg. 2007;6:640-3.

9. Bjerke HS. Síndrome de Boerhaave y lesiones barogénicas del esófago. Chest Surg Clin N Am. 1994;4:819-25.

10. Dayen $C$, Mishellany $H$, Hellmuth D. Rotura espontánea del esófago 0 síndrome de Boerhaave. Reporte de 3 casos y revisión de la literatura. Rev Mal Respir. 2000;18:537-40.

11. Goldstein LA, Thompson WR. Esophageal perforations: a 15 year experience. Am J Surg. 1982;143:495-503.

12. Bradley SL, Pairolero PC, Payne S, Gracey DR. Spontaneous rupture of the esophagus. Arch Surg. 1981;116:755-8.

13. Pate JW, Walker WA, Cole Jr FH, Owen EW, Jonson WH. Spontaneous rupture of the esophagus: a 30-year experience. Ann Thorac Surg. 1989;47:689-92.

14. Jagminas $L$, Silverman RA. Boerhaave's syndrome presenting with abdominal pain and right hydropneumothorax. Am J Emerg Med. 1996;14:53-56.

15. Moreno FC, Zamudio TA. Ruptura espontánea del esófago consecutiva a hematemesis por úlcera gástrica. Rev Gastroenterol Mex. 1975;40:3-7.

16. Walker WS, Cameron EWJ, Walbaum PR. Diagnosis and management of espontaneous transmural rupture of the oesophagus (Boerhaave's syndrome). Br J Surg. 1985;72:204-7.

17. Jones II WG, Ginsberg RJ. Esophageal perforation: a continuing challenge. Ann Thorac Surg. 1992;53:534-43.

18. Katabathina VS, Restrepo CS, Martínez-Jiménez S, Riascos RF. Nonvascular, nontraumatic mediastinal emergencies in adults: a comprehensive review of imaging findings. Radiographics. 2011;31:1141-60.

19. Folan RD, Smith RE, Head JM. Esophageal hematoma requiring emergency surgical intervention. Dig Dis Sci. 1992;37:1918-21.

20. Cherednikov EF, Kunin AA, Cherednikov EE, Moiseeva NS. The role of etiopathogenetic aspects in prediction and prevention of discontinuous-hemorrhagic (Mallory-Weiss) syndrome. EPMA J. 2016;7:7.

21. Van der Weg G, Wikkeling M, Van Leeuwen M, Ter Avest E. A rare case of oesophageal rupture: Boerhaave's syndrome. Int J Emerg Med. 2014;7:27.

22. Soreide JA, Viste A. Esophageal perforation: diagnostic work-up and clinical decision-making in the first 24 hours. Scand J Trauma Resusc Emerg Med. 2011;19:66.

23. Gupta NM, Kaman L. Personal management of 57 consecutive patients with esophageal perforation. Am J Surg. 2004;187:58-63.

24. Giménez A, Franquet T, Erasmus JJ, Martínez S, Estrada P. Thoracic complications of esophageal disorders. Radiographics 2002; 22 Spec No: S247-58.

25. Turner AR, Turner SD. Boerhaave syndrome. StatPearls. Treasure Island (FL): StatPearls Publishing; 2020.

26. Brinster JB, Singhal S, Lee L, Marshall MB, Kaiser LR, Kucharczuk JC. Evolving options in the management of esophageal perforation. Ann Thorac Surg. 2004;77:1475-83. 